\title{
On a possibility of exotic heavy baryons
}

\author{
Michal Praszalowicz* \\ Marian Smoluchowski Institute of Physics, Jagiellonian University, \\ Łojasiewicza 11, 30-348 Kraków, Poland \\ E-mail: michaleif.uj.edd.pl
}

\begin{abstract}
Triggered by a recent announcement by the $\mathrm{LHCb}$ Collaboration of five excited $\Omega_{c}^{0}$ resonances of small widths we propose an interpretation based on the Chiral Quark Soliton Model. We argue that three of the LHCb resonances are parity (-) excitations of the ground state SU(3) sextets, while the other two with the smallest widths are exotic pentaquarks that belong to the SU(3) $\overline{\mathbf{1 5}}$. We first briefly review the model and discuss the status of the putative $\Theta^{+}(1540)$. Next we show how to generalize the model to the case of heavy baryons. We test this approach against the ground state $\overline{\mathbf{3}}$ and $\mathbf{6}$ heavy baryons, and discuss different excitations that are possible in the Chiral Quark Soliton Model. We show that the model accommodates two excited $\overline{\mathbf{3}}$ in the charm sector that are experimentally observed. Finally we discuss possible assignments of the $\mathrm{LHCb} \Omega_{c}^{0}$ states.
\end{abstract}

Corfu Summer Institute 2017 'School and Workshops on Elementary Particle Physics and Gravity' 2-28 September 2017

Corfu, Greece

${ }^{*}$ Speaker. 


\section{Introduction}

In a recent paper the $\mathrm{LHCb}$ collaboration announced five, or even six $\Omega_{c}^{0}$ states with masses in the range of $3-3.2 \mathrm{GeV}$ [1]. These states have been later confirmed by BELLE [2]. In this report we summarize our recent works on heavy baryons [3, 4, 5] (see also [6, 7, 8]) where we have applied the Chiral Quark Soliton Model $(\chi \mathrm{QSM})$ to the baryonic systems with one heavy quark. This paper is based on Ref. [9], a shortened version of this report has already appeared [10].

The LHCb discovery triggered theoretical activity to interpret $\Omega_{c}^{0}$ 's in different approaches. These include QCD sum rules [12, 13, 14, 15, 17], constituent quark models [18], chiral quark models [19], lattice QCD [20] and holographic QCD [21]. In Refs. [22, 23, 24, 25] the new states are treated as bound states of a charm quark and a light diquark, the authors of Refs. [26, 27, $28,29]$ interpreted the new states as molecular states and in some approaches [30, 31, 32, 33] as pentaquarks. Regge trajectories approach has been put forward in Ref. [34]. Decay properties of these states have been studied in a ${ }^{3} P_{0}$ model [35], quark potential models [36], chiral quark model [37] and in molecular model [38]. Coupled channel approach to study the $\mathrm{LHCb} \Omega_{c}^{0}$ states has been applied in Refs. [39, 40]. For theoretical review of heavy exotica see Ref. [41] and for experimental overview Ref. [42].

Such a large number of possible theoretical interpretations may at first glance come as a surprise. Indeed, naive quark model seems to provide the simplest and successful picture. Let us recall that two light quarks may form SU(3) flavor $\overline{\mathbf{3}}$ and $\mathbf{6}$. Because of the Fermi exclusion principle $\overline{\mathbf{3}}$ has spin 0 and $\mathbf{6}$ spin 1 . Adding a heavy quark results in one $\mathrm{SU}(3)$ antitriplet of spin $1 / 2$ and two hyperfine split sextets of spin $1 / 2$ and $3 / 2$. The simplest possible excitation consists in adding the angular momentum to the system, which in the heavy quark rest frame maybe interpreted as the angular momentum of the light subsystem. Such configuration would have negative parity. An immediate consequence of this picture is the emergence of two hyper-fine split antitriplets of spin $1 / 2^{-}$and $3 / 2^{-}$that are indeed observed experimentally both in charm and (partially) bottom sectors. In the sextet case total angular momentum of the light subsystem can be 0,1 or 2 . Therefore one predicts five excited sextets of negative parity: two with total spin $1 / 2$, two with total spin $3 / 2$ and one with total spin $5 / 2$. The LHCb resonances would be the first experimentally observed particles from these multiplets. Unfortunately, when it comes to a more detailed analysis of the LHCb data, basically all models have problems to accommodate all five LHCb resonances within the above scenario with acceptable accuracy. Therefore an alternative assignments of some of the LHCb resonaces have been necessary.

We have encountered similar problems in the $\chi$ QSM. In a recent paper [3] we have shown that the ground state sextets together with the ground state $\overline{\mathbf{3}}$ that comprises $\Lambda_{c}(2280)$ and $\Xi_{c}(2470)$ can be successfully described in terms of the $\chi$ QSM supplemented by an interaction with a heavy quark in such a way that heavy quark symmetry [11] is respected. A great advantage of the $\chi$ QSM consists in a rather restrictive mass formula linking the spectra of light baryons with the heavy ones in question, which - as mentioned above - does not allow to accommodate all five LHCb $\Omega_{c}^{0}$ resonances in the parity (-) excitations of the ground state sextet. Therefore we have been forced to find an alternative assignment for some of these states. A natural interpretation within the framework of the $\chi \mathrm{QSM}$ is to associate two narrowest (with the decay width of the order of 1 $\mathrm{MeV}) \Omega_{c}^{0}$ 's with parity (+) exotic pentaquarks. 


\section{Chiral Quark Soliton Model for light baryons}

$\chi$ QSM [43] (for review see Refs. [44, 45, 46] and references therein) is based on an old argument by Witten [47], which says that in the limit of a large number of colors $\left(N_{c} \rightarrow \infty\right), N_{c}$ relativistic valence quarks generate chiral mean fields represented by a distortion of the Dirac sea that in turn interacts with the valence quarks themselves. The soliton configuration corresponds to the solution of the Dirac equation for the constituent quarks (with gluons integrated out) in the mean-field approximation where the mean fields respect so called hedgehog symmetry. Since it is impossible to construct a pseudoscalar field that changes sign under inversion of coordinates, which would be compatible with the $\mathrm{SU}(3)_{\text {flav }} \times \mathrm{SO}(3)$ space symmetry one has to resort to a smaller hedgehog symmetry that, however, leads to the correct baryon spectrum (see below). This means that neither spin $(\boldsymbol{S})$ nor isospin $(\boldsymbol{T})$ are good quantum numbers. Instead a grand $\operatorname{spin} \boldsymbol{K}=\boldsymbol{S}+\boldsymbol{T}$ is a good quantum number.

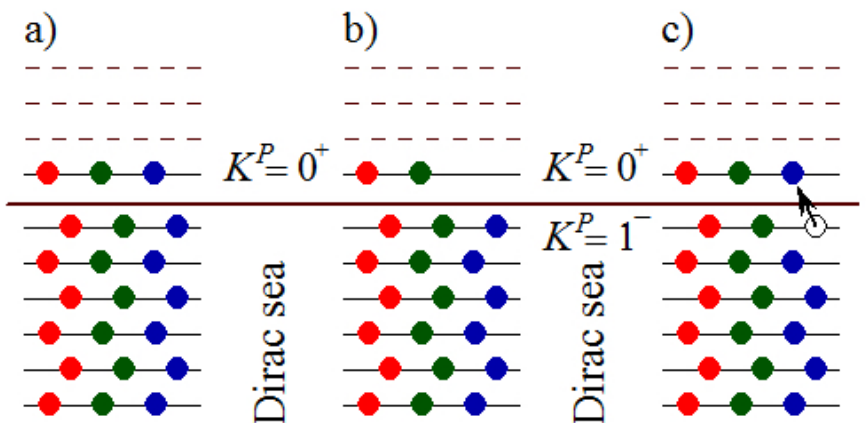

Figure 1: Schematic pattern of light quark levels in a self-consistent soliton configuration. In the left panel all sea levels are filled and $N_{c}$ (=3 in the Figure) valence quarks occupy the $K^{P}=0^{+}$lowest positive energy level. Unoccupied positive energy levels are dpicted by dashed lines. In the middle panel one valence quark has been stripped off, and the soliton has to be supplemented by a heavy quark not shown in the Figure. In the right panel a possible excitation of a sea level quark, conjectured to be $K^{P}=1^{-}$, to the valence level is shown, and again the soliton has to couple to a heavy quark. Strange quark levels that exhibit different filling pattern are not shown.

The ground state configuration corresponds to the fully occupied $K^{P}=0^{+}$valence level, as shown in Fig. 1.a. Therefore the soliton does not carry definite quantum numbers except for the baryon number resulting from the valence quarks. Spin and isospin appear when the rotations in space and flavor are quantized and the resulting collective hamiltonian analogous to the one of a symmetric top is computed. There are two conditions that the collective wave functions have to satisfy:

- allowed SU(3) representations must contain states with hypercharge $Y^{\prime}=N_{c} / 3$,

- the isospin $\boldsymbol{T}^{\prime}$ of the states with $Y^{\prime}=N_{c} / 3$ couples with the soliton spin $\boldsymbol{J}$ to a singlet: $\boldsymbol{T}^{\prime}+\boldsymbol{J}=0$

In the case of light positive parity baryons the lowest allowed representations are 8 of spin $1 / 2$, $\mathbf{1 0}$ of spin $3 / 2$, and also exotic $\overline{\mathbf{1 0}}$ of spin $1 / 2$ with the lightest state corresponding to the putative 


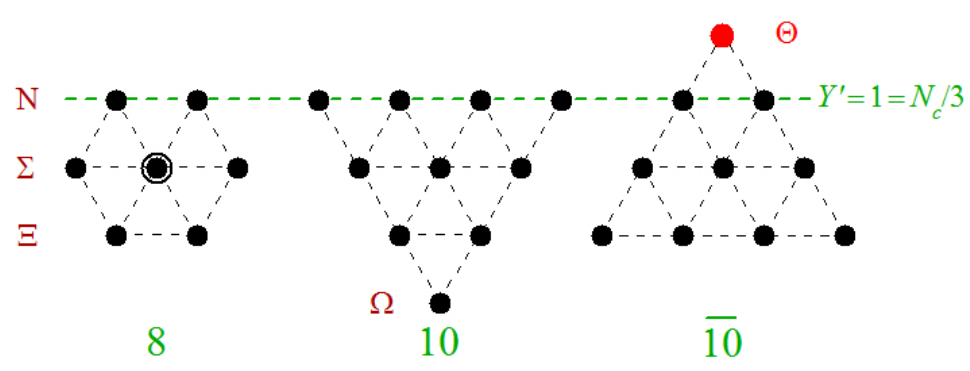

Figure 2: Lowest lying SU(3) flavor representations allowed by the constraint $Y^{\prime}=1$. The first exotic representation, $\overline{\mathbf{1 0}}$, contains the explicitly exotic pentaquark states $\Theta^{+}, \Xi^{+}$and $\Xi^{-}$and non-exotic nucleonand sigma-like states.

$\Theta^{+}(1540)[48,49]$. They are shown in Fig. 2. Chiral models in general predict that pentaquarks are light $[48,49]$ and - in some specific models - narrow [49].

After the first enthusiastic announcements of the discovery of pentaquarks in 2003 by LEPS [50] and DIANA [51] collaborations, the experimental evidence for the light exotica has been questioned (see e.g. [52]). Nevertheless, both DIANA [53] and LEPS [54] upheld their original claims after performing higher statistics analyses. The report on exotic $\Xi$ states (see Fig. 2) by NA49 [55] from 2004, to the best of my knowledge, has not been questioned so far, however the confirmation is still strongly needed.

Another piece of information on $\overline{\mathbf{1 0}}$ comes from the $\eta$ photo-production off the nucleon. Different experiments confirm the narrow structure at the c.m.s. energy $W \sim 1.68 \mathrm{GeV}$ observed in the case of the neutron, whereas no structure is observed on the proton (see Fig. 27 in the latest report by CBELSA/TAPS Collaboration [56] and references therein). The natural interpretation of this "neutron puzzle" was proposed already in 2003 in Ref. [57]. There one assumes that the narrow excitation at $W \sim 1.68 \mathrm{GeV}$ corresponds to the non-exotic penta-nucleon resonance belonging to $\overline{\mathbf{1 0}}$. Indeed, the $\mathrm{SU}(3)$ symmetry forbids photo-excitation of the proton member of $\overline{\mathbf{1 0}}$, while the analogous transition on the neutron is possible. This is due to the fact that photon is an $\operatorname{SU}(3)$ $U$-spin singlet, and the $U$-spin symmetry is exact in the $\mathrm{SU}(3)$ symmetric limit. An alternative interpretation is based on a partial wave analysis in terms of the Bonn-Gatchina approach [58]. There is an ongoing dispute on the interpretation of the "neutron puzzle" (for the latest arguments see Ref. [59]).

\section{Ground state heavy baryons}

Recently wa have proposed [3], following Ref. [60], to generalize the above approach to heavy baryons, by stripping off one valence quark from the $K^{P}=0^{+}$level, as shown in Fig. 1.b, and replacing it by a heavy quark to neutralize the color. In the large $N_{c}$ limit both systems: light and heavy baryons are described essentially by the same mean field, and the only difference is now in the quantization condition:

- allowed SU(3) representations must contain states with hypercharge $Y^{\prime}=\left(N_{c}-1\right) / 3$.

The lowest allowed SU(3) representations are in this case (as in the quark model) $\overline{\mathbf{3}}$ of spin 0 and to $\mathbf{6}$ of spin 1 shown in Fig. 3. Therefore, the baryons constructed from such a soliton and a heavy 
quark form an $\mathrm{SU}(3)$ antitriplet of spin $1 / 2$ and two sextets of spin $1 / 2$ and $3 / 2$ that are subject to a hyper-fine splitting.

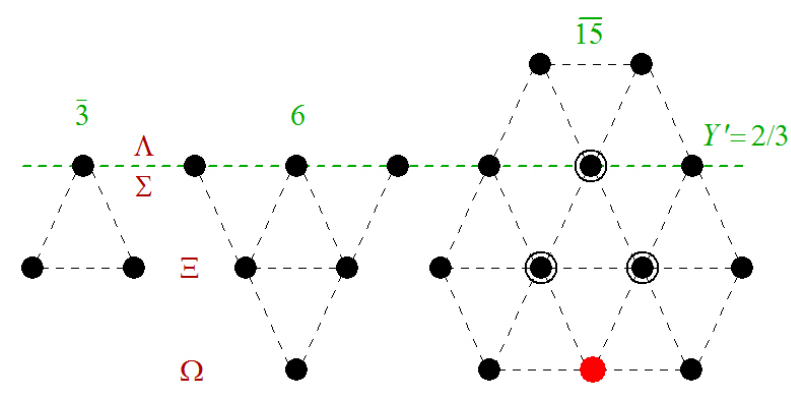

Figure 3: Rotational band of a soliton with one valence quark stripped off. Soliton spin corresponds to the isospin $T^{\prime}$ of states on the quantization line $Y^{\prime}=2 / 3$. We show three lowest allowed representations: antitriplet of spin 0 , sextet of spin 1 and the lowest exotic representation $\overline{\mathbf{1 5}}$ of spin 1 or 0 . Heavy quark has to be added.

It is interesting to look at the so called model-independent relations ${ }^{1}$ generated by the symmetries of the $\chi$ QSM that include Gell-Mann-Okubo SU(3) mass relations within given baryon multiplet, and the so called Guadaginini [62] relations that follow from the hedgehog symmetry and relate mass splittings between different multiplets. The model predicts equal mass splittings separately in the $\overline{\mathbf{3}}$ and $\mathbf{6}$, which are proportional to the hypercharge $Y$

$$
\Delta M=\delta_{\overline{3}, 6} \times Y
$$

that are independent of the spin and of the heavy quark mass. These relations are indeed very well satisfied. For the $\overline{\mathbf{3}}$ we have (in $\mathrm{MeV}$ ):

$$
-\delta_{\overline{3}}=182.9 \pm\left. 0.3\right|_{\Xi_{c}-\Lambda_{c}}=173.6 \pm\left. 0.7\right|_{\Xi_{b}-\Lambda_{b}},
$$

which is satisfied with $7 \%$ accuracy. In the case of the 6 we have more relations (in MeV):

$$
\begin{aligned}
-\delta_{6} & =123.3 \pm\left. 2.1\right|_{\Xi_{c}^{\prime}-\Sigma_{c}}=118.4 \pm\left. 2.7\right|_{\Omega_{c}-\Xi_{c}^{\prime}} \\
& =127.8 \pm\left. 0.8\right|_{\Xi_{c}^{*}-\Sigma_{c}^{*}}=120.0 \pm\left. 2.0\right|_{\Omega_{c}^{*}-\Xi_{c}^{*}} \\
& =121.6 \pm\left. 1.3\right|_{\Xi_{b}^{\prime}-\Sigma_{b}}=113.0 \pm\left. 1.9\right|_{\Omega_{b}-\Xi_{b}^{\prime}} \\
& =121.7 \pm\left. 1.3\right|_{\Xi_{b}^{*}-\Sigma_{b}^{*}} .
\end{aligned}
$$

We see that the equality of splittings is quite accurate (at the $6 \%$ level). On the other hand the model predicts the values of the splitting parameters [3] using as an input mass splittings of the light baryons:

$$
\delta_{\overline{3}}=-203.8 \pm 3.5, \quad \delta_{6}=-135.2 \pm 3.3,
$$

in units of $\mathrm{MeV}$, which overestimate the model-independent determination $(3.1,3.2)$ by approximately $13 \%$. This is entirely within the expected accuracy of this approach.

\footnotetext{
${ }^{1}$ The term model-independent relations in the present context has been first used in Ref. [61].
} 
In order to remove the degeneracy between sextet spin $1 / 2$ and $3 / 2$ multiplets, we introduce the spin-spin interaction hamiltonian expressed as:

$$
H_{L Q}=\frac{2}{3} \frac{\kappa}{m_{Q} M_{\mathrm{sol}}} \boldsymbol{J} \cdot \boldsymbol{S}_{Q}=\frac{2}{3} \frac{\varkappa}{m_{Q}} \boldsymbol{J} \cdot \boldsymbol{S}_{Q}
$$

where $\varkappa$ denotes the flavor-independent hyperfine coupling. The operators $\boldsymbol{J}$ and $\boldsymbol{S}_{Q}$ represent the spin operators for the soliton and the heavy quark, respectively. $M_{\text {sol }}$ has been incorporated into an unknown coefficient $\varkappa$. The Hamiltonian $H_{L Q}$ does not affect the $\overline{3}$ states, since in this case $J=0$. In $6 J=1$, and it couples to $S_{Q}$ producing two multiplets $S=1 / 2$ and $S=3 / 2$.

We arrive therefore at another set of model-independent relations, which are not directly related to the specifics of the soliton model, but provide a test of our assumption concerning the spin interactions of Eq. (3.4):

$$
\begin{aligned}
& \frac{\varkappa}{m_{c}}=64.5 \pm\left. 0.8\right|_{\Sigma_{c}}=69.1 \pm\left. 2.1\right|_{\Xi_{c}}=70.7 \pm\left. 2.6\right|_{\Omega_{c}} \\
& \frac{\varkappa}{m_{b}}=20.2 \pm\left. 1.9\right|_{\Sigma_{b}}=20.3 \pm\left. 0.1\right|_{\Xi_{b}} .
\end{aligned}
$$

(in $\mathrm{MeV}$ ). From the ratios of the spin splittings (3.5) we can determine the ratio of the heavy-quark masses

$$
\frac{m_{c}}{m_{b}}=0.29-0.31
$$

The experimental values of the $\overline{\mathrm{MS}}$ heavy quark masses lead to $m_{c} / m_{b}=0.305$ where both masses $m_{Q}$ are evaluated at the renormalization point $\mu=m_{Q}$ [63].

Apart from equal splittings in 6 (3.2), the model admits a sum rule relating particles from two spin $1 / 2$ and spin $3 / 2$ sextets (the latter denoted with an asterisk):

$$
M_{\Omega_{Q}^{*}}=2 M_{\Xi_{Q}^{\prime}}+M_{\Sigma_{Q}^{*}}-2 M_{\Sigma_{Q}}
$$

Equation (3.7) yields $(2764.5 \pm 3.1) \mathrm{MeV}$ for $M_{\Omega_{c}^{*}}$, which is $1.4 \mathrm{MeV}$ below the experiment, and predicts

$$
M_{\Omega_{b}^{*}}=(6076.8 \pm 2.25) \mathrm{MeV}
$$

for yet unmeasured $\Omega_{b}^{*}$.

Another phenomenological test of the model consists in the calculation of the decay widths. Indeed, using heavy baryon chiral perturbation theory [64] and the Goldberger-Treiman relation [65] one can derive the formulae for the $p$-wave strong decay widths of the ground state sextets:

$$
\begin{aligned}
& \Gamma_{\Sigma\left(6_{1}\right) \rightarrow \Lambda\left(\overline{3}_{0}\right)+\pi}=\frac{1}{72 \pi} \frac{p^{3}}{F_{\pi}^{2}} \frac{M_{\Lambda\left(\overline{3}_{0}\right)}}{M_{\Sigma\left(6_{1}\right)}} H_{3} \frac{3}{8}, \\
& \Gamma_{\Xi\left(6_{1}\right) \rightarrow \Xi\left(\overline{3}_{0}\right)+\pi}=\frac{1}{72 \pi} \frac{p^{3}}{F_{\pi}^{2}} \frac{M_{\Xi\left(\overline{3}_{0}\right)}}{M_{\Xi\left(6_{1}\right)}} H_{\frac{2}{3}} \frac{9}{32}
\end{aligned}
$$

where

$$
H_{\overline{3}}=-a_{1}+\frac{1}{2} a_{2}
$$


Constants $a_{i}$ enter the definition of the axial-vector current ${ }^{2}$ and have been extracted from the semileptonic decays of the baryon octet in Ref. [66]. Model predictions (which are parameter free with one modification related to the rescaling of $a_{1}$ due to the fact that contrary to the light sector we have $N_{c}-1$ valence quarks rather than $N_{c}$ ) for the partial decay widths are compared with the experimental data in Fig. 4. Surprisingly good agreement between predicted decay widths and the experimental data encourages us to apply the model to the excited heavy baryons.
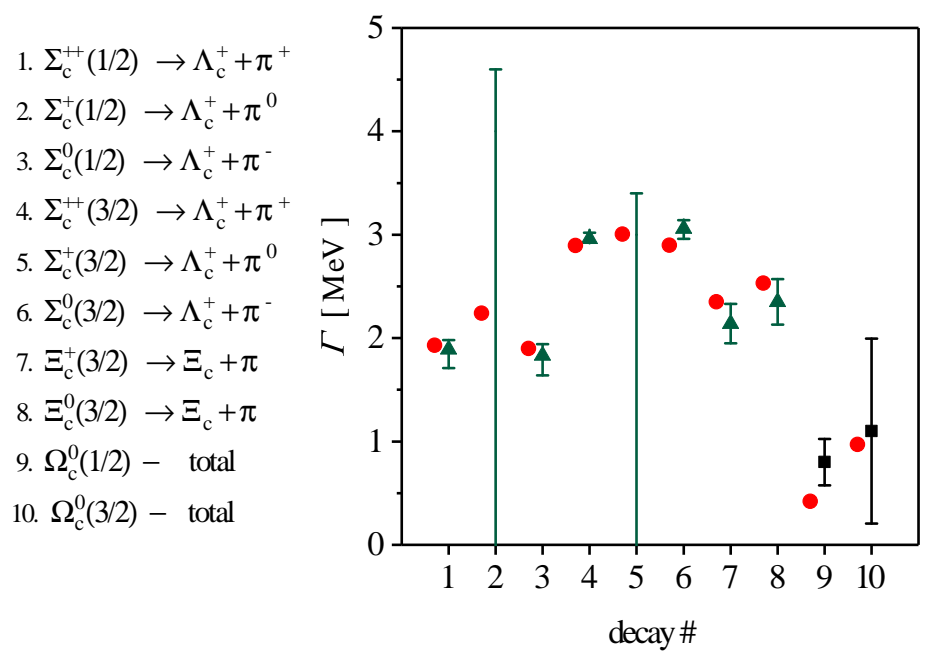

Figure 4: Decay widths of the charm baryons. Red full circles correspond to our theoretical predictions. Dark green triangles correspond to the experimental data [63]. Data for decays $4-6$ of $\Sigma_{c}\left(6_{1}, 3 / 2\right)$ have been divided by a factor of 5 to fit within the plot area. Widths of two LHCb [1] $\Omega_{c}^{0}$ states that we interpret as pentaquarks are plotted as black full squares with theoretical values shown as red full circles.

\section{Excited heavy baryons}

Two possible kinds of excitations are present in the $\chi$ QSM. Firstly, higher SU(3) representations, similar to the antidecuplet in the light sector, appear in the rotational band of the soliton of Fig. 1.b. The lowest possible exotic SU(3) representation is $\overline{\mathbf{1 5}}$ of positive parity and spin $1(\overline{\mathbf{1 5}}$ of spin 0 is heavier) depicted in Fig. 3. Second possibility corresponds to the excitation of the sea quark from the $K^{P}=1^{-}$sea level to the valence level [60] shown in Fig. 1.c (or alternatively valence quark excitation to the first excited level ${ }^{3}$ of $K^{P}=1^{-}$). In this case the parity is negative but the rotational band is the same as in Fig. 3 with, however, different quantization condition:

- the isospin $\boldsymbol{T}^{\prime}$ of the states with $Y^{\prime}=\left(N_{c}-1\right) / 3$ couples with the soliton spin $\boldsymbol{J}$ as follows: $\boldsymbol{T}^{\prime}+\boldsymbol{J}=\boldsymbol{K}$, where $\boldsymbol{K}$ is the grand spin of the excited level.

The first allowed SU(3) representation for one quark excited soliton is again $\overline{\mathbf{3}}$ with $T^{\prime}=0$, which - according to the above condition for $K=1$ is quantized as spin $J=1$. We therefore expect

\footnotetext{
${ }^{2}$ For the reader's convenience we give the relations of the constants $a_{1,2,3}$ to nucleon axial charges in the chiral limit: $g_{A}=\frac{7}{30}\left(-a_{1}+\frac{1}{2} a_{2}+\frac{1}{14} a_{3}\right), g_{A}^{(0)}=\frac{1}{2} a_{3}, g_{A}^{(8)}=\frac{1}{10 \sqrt{3}}\left(-a_{1}+\frac{1}{2} a_{2}+\frac{1}{2} a_{3}\right)$

${ }^{3}$ We thank Victor Petrov for pointing out this possibility.
} 
two hyper-fine split anti-triplets of spin $1 / 2$ and 3/2. For each of them the $m_{s}$ splitting parameter $\delta_{\overline{\mathbf{3}}}^{\prime}$ is given by the same formula as for the ground state antitriplet, and therefore we know its numerical value [3]:

$$
\delta_{\mathbf{3}}^{\prime}=\delta_{\overline{\mathbf{3}}}=-180 \mathrm{MeV} .
$$

There are rather well measured candidates for both anti-triplets: for $(1 / 2)^{-}$we have $\Lambda_{c}(2592)$ and $\Xi_{c}(2790)$ and for $(3 / 2)^{-}$there exist $\Lambda_{c}(2628)$ and $\Xi_{c}(2818)$. From this assignment we get $\delta_{\overline{3}}^{\prime}=$ $-198 \mathrm{MeV}$ and $-190 \mathrm{MeV}$ respectively, in relatively good agreement with Eq.(4.1). Furthermore, we can extract hyper-fine splitting parameter:

$$
\frac{\kappa^{\prime}}{m_{c}}=30 \mathrm{MeV}
$$

which is different from Eq. (3.5) since it corresponds to a different soliton configuration, namely the one of Fig. 1.c

Next negative parity excitation is the SU(3) sextet (with $T^{\prime}=1$, see Fig. 3), which for $K=1$ gives the soliton spin $J=0,1,2$ in close analogy to the total angular momentum of light subsystem in the quark model. By adding one heavy quark we end up with five possible total spin $S$ excitations: for $J=0: S=1 / 2$, for $J=1: S=1 / 2$ and $3 / 2$, and for $J=2: S=3 / 2$ and $5 / 2$. The $m_{s}$ mass splittings depend in this case on $J$ and read

$$
\delta_{6}^{\prime}=\delta_{6}-\frac{3}{20} \delta \times\left\{\begin{aligned}
& 2 \text { for } J=0 \\
& 1 \text { for } J=1 \\
&-1 \text { for } J=2
\end{aligned}\right.
$$

where $\delta_{6}=-120 \mathrm{MeV}$ [3] corresponds to the ground state sextet splitting. Unfortunately we do not know the value of a new parameter $\delta$ corresponding to the quark transition depicted in Fig. 1.c, which is absent in the case of light baryons. It is however possible to eliminate the unknown parameters (not only $\delta$ but also another parameter in the rotational hamiltonian) and obtain spectrum of the $\Omega_{Q}$ members of the excited sextets depicted in Fig. 5. We have checked in Ref. [4] that it is impossible to fit all five LHCb states into the pattern of Fig. 5.

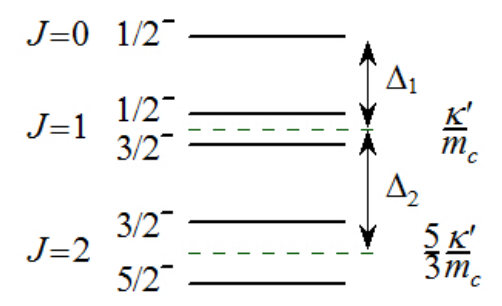

Figure 5: Schematic spectrum of the $\Omega_{Q}$ members of the excited sextets.

We have therefore forced model constraints of Fig. 5, which allows to accommodate only three out of five LHCb states (see black vertical lines in Fig. 6). Two heaviest $\chi$ QSM states (green lines in Fig. 6) lie already above the decay threshold to heavy mesons, and it is quite possible that they have very small branching ratio to the $\Xi_{c}^{+}+K^{-}$final state analyzed by the LHCb. Two remaining states indicated by dark-blue arrows in Fig. 6, which are hyper fine split by $70 \mathrm{MeV}$ (as the ground state sextets that belong to the same rotational band, see the first line of Eq. (3.5)), can be therefore 
interpreted as the members of exotic $\overline{\mathbf{1 5}}$ of positive parity shown as a red dot in Fig. 3. This interpretation is reinforced by the decay widths, which can be computed in the model:

$$
\begin{aligned}
& \Gamma_{\Omega\left(\overline{15}_{1}\right) \rightarrow \Xi\left(\overline{3}_{0}\right)+K}=\frac{1}{72 \pi} \frac{p^{3}}{F_{K}^{2}} \frac{M_{\Xi\left(\overline{3}_{0}\right)}}{\left.M_{\Omega(\overline{15})}\right)} G_{\overline{3}} \frac{3}{10}, \\
& \Gamma_{\Omega\left(\overline{15}_{1}\right) \rightarrow \Omega\left(6_{1}\right)+\pi}=\frac{1}{72 \pi} \frac{p^{3}}{F_{\pi}^{2}} \frac{M_{\Omega\left(6_{1}\right)}}{M_{\Omega\left(\overline{15}_{1}\right)}} G_{6}^{2} \frac{4}{15} \gamma, \\
& \Gamma_{\Omega\left(\overline{15}_{1}\right) \rightarrow \Xi\left(6_{1}\right)+K}=\frac{1}{72 \pi} \frac{p^{3}}{F_{K}^{2}} \frac{M_{\Xi\left(6_{1}\right)}}{M_{\Omega\left(\overline{15}_{1}\right)}} G_{6}^{2} \frac{2}{15} \gamma
\end{aligned}
$$

where coefficients $\gamma$ depend on the initial and final spin of the involved baryons:

$$
\begin{array}{ll}
\gamma(1 / 2 \rightarrow 1 / 2)=2 / 3, & \gamma(1 / 2 \rightarrow 3 / 2)=1 / 3, \\
\gamma(3 / 2 \rightarrow 1 / 2)=1 / 6, & \gamma(3 / 2 \rightarrow 3 / 2)=5 / 6 .
\end{array}
$$

Here

$$
\begin{aligned}
& G_{\overline{3}}=-a_{1}-\frac{1}{2} a_{2}, \\
& G_{6}=-a_{1}-\frac{1}{2} a_{2}-a_{3} .
\end{aligned}
$$

These widths are of the order of $1 \mathrm{MeV}$ and agree with the LHCb measurement (see Fig. 4). Such small widths are in fact expected in the present approach, since the leading $N_{c}$ terms of the couplings $G_{\overline{3}}$ and $G_{6}$ cancel in the non-relativistic limit.

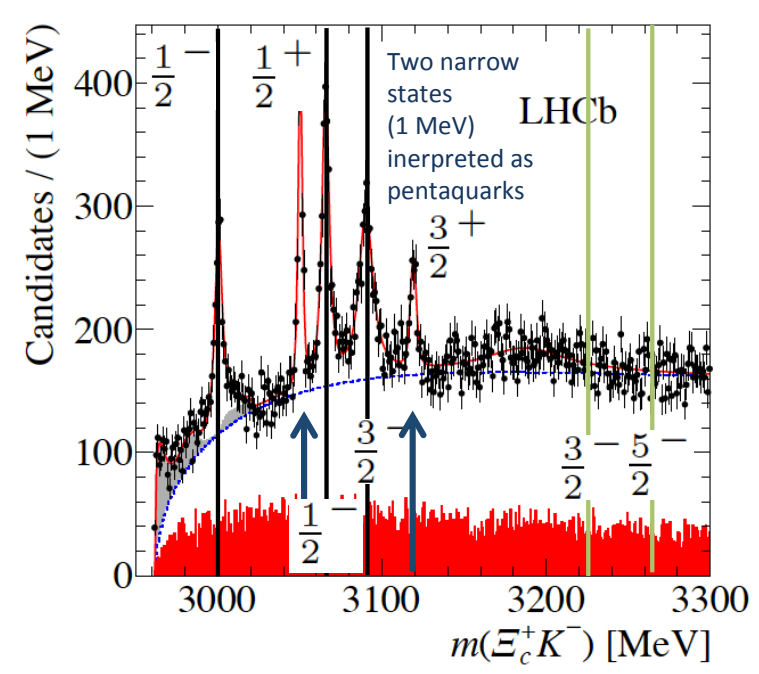

Figure 6: Spectrum of the $\Omega_{c}^{0}$ states (from Ref.[8]) with theoretical predictions of the present model

\section{Summary}

In the large $N_{c}$ limit both heavy and light baryons are described by the universal mean-field. This allows us to relate the properties of heavy baryons to the light ones. We have shown in 
Ref. [3] that the universal mean field gives simultaneously good description of the ground-state $\overline{\mathbf{3}}$ and 6 multiplets of heavy baryons. In Ref. [4] we have demonstrated that the same picture predicts the following excited states for heavy-quark baryons:

- two hyper-fine split $\left(1 / 2^{-}\right.$and $\left.3 / 2^{-}\right) \overline{\mathbf{3}}^{\prime}$ which experimentally have very good candidates,

- five excited sexstets (rotationally and hyper-fine split) with quantum numbers $\left(J=0,1 / 2^{-}\right)$, $\left(J=1,1 / 2^{-}, 3 / 2^{-}\right)$and $\left(J=2,3 / 2^{-}, 5 / 2^{-}\right)$, where $J$ denotes the soliton spin,

- two hyper-fine split exotic $\overline{\mathbf{1 5}}$-plets with quantum numbers $1 / 2^{+}$and $3 / 2^{+}$.

The observation of the new excited $\Omega_{c}^{0}$ 's allows us to get insight into the excited sextets and $\overline{\mathbf{1 5}}$-plets. We identify the observed $\Omega_{c}(3000), \Omega_{c}(3066)$ and $\Omega_{c}(3090)$ with $\left(J=0: 1 / 2^{-}\right)$ and $\left(J=1: 1 / 2^{-}, 3 / 2^{-}\right)$states from the excited sextet, whereas the most narrow $\Omega_{c}(3050)$ and $\Omega_{c}(3119)$ states we identify with $\left(J=1: 1 / 2^{+}, 3 / 2^{+}\right)$states from the exotic $\overline{\mathbf{1 5}}$ multiplet. The remaining two $\left(J=2: 3 / 2^{-}, 5 / 2^{-}\right)$states from the sextet have masses above the $\Xi+D$ threshold $(3185 \mathrm{MeV})$, so they are probably hidden in a large bump observed by the LHCb collaboration above $3200 \mathrm{MeV}$. It should be stressed that the simplest scenario in which all five LHCb $\Omega_{c}^{0}$ states are classified as members of the excited sextets contradicts general mass formulae derived within the $\chi \mathrm{QSM}$.

The simplest way to confirm or falsify our identification is to search for the isospin partners of $\Omega_{c}^{0}$ from the $\overline{\mathbf{1 5}}$. For example, they can be searched in the mass distribution of $\Xi_{c}^{0}+K^{-}$or $\Xi_{c}^{+}+\bar{K}^{0}$, the $\Omega_{c}^{0}$ 's from the sextet do not decay into these channels.

\section{Acknowledgments}

It is a pleasure to thank H.-C. Kim, M.V. Polyakov and G.S. Yang for a fruitful collaboration that led to the research reported in this paper. I would like to thank the organizers of the Corfu Summer Institute 2017 'School and Workshops on Elementary Particle Physics and Gravity' where these results were presented, for very stimulating and interesting meeting. This work was supported by the Polish NCN grant 2017/27/B/ST2/01314.

\section{References}

[1] R. Aaij et al. [LHCb Collaboration], Phys. Rev. Lett. 118 (2017) 182001.

[2] J. Yelton et al. [Belle Collaboration], Phys. Rev. D 97 (2018) 051102.

[3] G. S. Yang, H. C. Kim, M. V. Polyakov and M. Praszalowicz, Phys. Rev. D 94 (2016) 071502.

[4] H. C. Kim, M. V. Polyakov and M. Praszalowicz, Phys. Rev. D 96 (2017) 014009, Addendum: [Phys. Rev. D 96 (2017) 039902].

[5] H. C. Kim, M. V. Polyakov, M. Praszalowicz and G. S. Yang, Phys. Rev. D 96 (2017) 094021, Erratum: [Phys. Rev. D 97 (2018) 039901].

[6] J. Y. Kim, H. C. Kim and G. S. Yang, arXiv:1801.09405 [hep-ph].

[7] G. S. Yang and H. C. Kim, Phys. Lett. B 781 (2018) 601. 
[8] J. Y. Kim and H. C. Kim, arXiv:1803.04069 [hep-ph].

[9] M. Praszalowicz, Acta Phys. Polon. B 48 (2017) 1775.

[10] M. Praszalowicz, arXiv:1805.03862 [hep-ph].

[11] N. Isgur and M. B. Wise, Phys. Lett. B 232 (1989) 113 and Phys. Rev. Lett. 66 (1991) 1130.

[12] Z. G. Wang, Eur. Phys. J. C 77 (2017) 325 and Z. G. Wang, X. N. Wei and Z. H. Yan, Eur. Phys. J. C 77 (2017) 832.

[13] H. X. Chen, Q. Mao, W. Chen, A. Hosaka, X. Liu and S. L. Zhu, Phys. Rev. D 95 (2017) 094008.

[14] S. S. Agaev, K. Azizi and H. Sundu, EPL 118 (2017) 61001 and Eur. Phys. J. C 77 (2017) 395 and S. S. Agaev, K. Azizi and H. Sundu, Phys. Rev. D 96 (2017) 094011.

[15] T. M. Aliev, S. Bilmis and M. Savci, arXiv:1704.03439 [hep-ph] and arXiv:1805.02964 [hep-ph].

[16] Q. Mao, H. X. Chen, A. Hosaka, X. Liu and S. L. Zhu, Phys. Rev. D 96 (2017) no.7, 074021 doi:10.1103/PhysRevD.96.074021 [arXiv:1707.03712 [hep-ph]].

[17] Q. Mao, H. X. Chen, A. Hosaka, X. Liu and S. L. Zhu, Phys. Rev. D 96 (2017) 074021.

[18] K. L. Wang, L. Y. Xiao, X. H. Zhong and Q. Zhao, Phys. Rev. D 95 (2017) 116010.

[19] G. Yang, J. Ping and J. Segovia, arXiv:1709.09315 [hep-ph].

[20] M. Padmanath and N. Mathur, Phys. Rev. Lett. 119 (2017) 042001.

[21] Y. Liu and I. Zahed, Phys. Rev. D 95 (2017) 116012 and Phys. Rev. D 96 (2017) 056027.

[22] M. Karliner and J. L. Rosner, Phys. Rev. D 95 (2017) 114012.

[23] W. Wang and R. L. Zhu, Phys. Rev. D 96 (2017) 014024.

[24] H. Y. Cheng and C. W. Chiang, Phys. Rev. D 95 (2017) 094018.

[25] A. Ali, L. Maiani, A. V. Borisov, I. Ahmed, M. Jamil Aslam, A. Y. Parkhomenko, A. D. Polosa and A. Rehman, Eur. Phys. J. C 78 (2018) 29.

[26] H. Huang, J. Ping and F. Wang, Phys. Rev. D 97 (2018) 034027.

[27] G. Montaña, A. Feijoo and À. Ramos, Eur. Phys. J. A 54 (2018) 64.

[28] C. Wang, L. L. Liu, X. W. Kang and X. H. Guo, arXiv:1710.10850 [hep-ph].

[29] R. Chen, A. Hosaka and X. Liu, Phys. Rev. D 97 (2018) 036016.

[30] G. Yang and J. Ping, Phys. Rev. D 97 (2018) 034023.

[31] C. S. An and H. Chen, Phys. Rev. D 96 (2017) 034012.

[32] V. V. Anisovich, M. A. Matveev, J. Nyiri and A. N. Semenova, Mod. Phys. Lett. A 32 (2017) 1750154.

[33] Z. G. Wang and J. X. Zhang, arXiv:1804.06195 [hep-ph].

[34] K. Chen, Y. Dong, X. Liu, Q. F. Lü and T. Matsuki, Eur. Phys. J. C 78 (2018) 20.

[35] Z. Zhao, D. D. Ye and A. Zhang, Phys. Rev. D 95 (2017) 114024.

[36] B. Chen and X. Liu, Phys. Rev. D 96 (2017) 094015.

[37] K. L. Wang, Y. X. Yao, X. H. Zhong and Q. Zhao, Phys. Rev. D 96 (2017) 116016.

[38] Y. Huang, C. j. Xiao, Q. F. Lü, R. Wang, J. He and L. Geng, arXiv:1801.03598 [hep-ph]. 
[39] V. R. Debastiani, J. M. Dias, W. H. Liang and E. Oset, arXiv:1710.04231 [hep-ph].

[40] J. Nieves, R. Pavao and L. Tolos, Eur. Phys. J. C 78 (2018) 114.

[41] A. Ali, J. S. Lange and S. Stone, Prog. Part. Nucl. Phys. 97 (2017) 123.

[42] S. L. Olsen, T. Skwarnicki and D. Zieminska, Rev. Mod. Phys. 90 (2018) 015003.

[43] D. Diakonov, V. Y. Petrov and P. V. Pobylitsa, Nucl. Phys. B 306 (1988) 809.

[44] C. V. Christov, A. Blotz, H. C. Kim, P. Pobylitsa, T. Watabe, T. Meissner, E. Ruiz Arriola and K. Goeke, Prog. Part. Nucl. Phys. 37 (1996) 91.

[45] R. Alkofer, H. Reinhardt and H. Weigel, Phys. Rept. 265 (1996) 139.

[46] V. Petrov, Acta Phys. Polon. B 47 (2016) 59.

[47] E. Witten, Nucl. Phys. B 160 (1979) 57, and 223 (1983) 422, and 223 (1983) 433.

[48] M. Praszalowicz, in Skyrmions and Anomalies: proceedings. Edited by M. Jezabek and M. Praszalowicz. Singapore, World Scientific, 1987. p. 112-131, and Phys. Lett. B 575 (2003) 234.

[49] D. Diakonov, V. Petrov and M. V. Polyakov, Z. Phys. A 359 (1997) 305.

[50] T. Nakano et al. [LEPS Collaboration], Phys. Rev. Lett. 91 (2003) 012002.

[51] V. V. Barmin et al. [DIANA Collaboration], Phys. Atom. Nucl. 66 (2003) 1715 [Yad. Fiz. 66 (2003) 1763].

[52] K. H. Hicks, Eur. Phys. J. H 37 (2012) 1.

[53] V. V. Barmin et al. [DIANA Collaboration], Phys. Rev. C 89 (2014) no.4, 045204.

[54] T. Nakano [LEPS and LEPS2 Collaborations], JPS Conf. Proc. 13 (2017) 010007.

[55] C. Alt et al. [NA49 Collaboration], Phys. Rev. Lett. 92 (2004) 042003.

[56] L. Witthauer et al. [CBELSA/TAPS Collaboration], Eur. Phys. J. A 53 (2017) no.3, 58.

[57] M. V. Polyakov and A. Rathke, Eur. Phys. J. A 18 (2003) 691.

[58] A. V. Anisovich, V. Burkert, E. Klempt, V. A. Nikonov, A. V. Sarantsev and U. Thoma, Phys. Rev. C 95 (2017) no.3, 035211.

[59] V. Kuznetsov et al., JETP Lett. 105 (2017) 625.

[60] D. Diakonov, Prog. Theor. Phys. Suppl. 186 (2010) 99.

[61] G. S. Adkins and C. R. Nappi, Nucl. Phys. B 249 (1985) 507.

[62] E. Guadagnini, Nucl. Phys. B 236 (1984) 35.

[63] C. Patrignani et al. (Particle Data Group), Chin. Phys. C 40 (2016) 100001.

[64] H. Y. Cheng and C. K. Chua, Phys. Rev. D 75 (2007) 014006 and Phys. Rev. D 92 (2015) 074014.

[65] T. M. Yan, H. Y. Cheng, C. Y. Cheung, G. L. Lin, Y. C. Lin and H. L. Yu, Phys. Rev. D 46 (1992) 1148 ; Erratum: Phys. Rev. D 55 (1997) 5851.

[66] G. S. Yang and H.-Ch. Kim, Phys. Rev. C 92 (2015) 035206. 